\title{
Fatty Acid Esterification with Polyols over Acidic Montmorillonite
}

\author{
Asma Chaari, Soumaya Bouguerra Neji* and Mouhamed Hedi Frikha \\ Laboratory of Natural Substances, Faculty of Sciences, University of Sfax, Route de Soukra Km 3, 5-3038- Sfax. TUNISIA
}

\begin{abstract}
The production of fatty acid esters from stearic, oleic, and palmitic acids and polyols (ethylene glycol and glycerol) was investigated in this work. A series of montmorillonite-based clays catalysts (KSF, KSF/0, KP10, and K10), having different physicochemical properties, were used as acidic catalysts. The influence of the specific surface area and the acidity of the catalysts on the esterification rate were explored. The best catalytic activities were obtained with KSF catalyst. The optimization of various factors on the reaction was also studied, including catalyst concentration, reaction temperature and molar ratio (polyol / fatty acid). The yield rate reached $94 \%$ under the optimum conditions and the recovery rate maintained more than $96 \%$ after 5 batches.
\end{abstract}

Key words: esterification, montmorillonite, clays, fatty acids, polyols

\section{INTRODUCTION}

Esters of alcohols and fatty acids have a variety of applications in industry such as in food and cosmetic, in flavors and fragrances, and as components or intermediates for products. Polyol-fatty acid esters are valuable chemical compounds that find application in pharmaceuticals, food and cosmetic production. They are also used as raw material for emulsifiers, lubricants and polymers ${ }^{1)}$.

Esterification is carried out in the homogenous phase in the presence of acid catalysts namely sulfuric acid, hydrochloric acid and p-toluenesulfonic acid (p-TsOH). The use of such catalysts has many inconvenients that include corrosion, loss of catalyst and environmental problems ${ }^{2,3)}$. A number of solid acids such as ion-exchange resin ${ }^{4}$, zeolite $^{5)}$, Tin-Organic Framework ${ }^{6)}$, Fe-Zn double-metal cyanide $(\mathrm{DMC})^{7)}$, zirconium phenyl phosphonate phosphite $(\mathrm{ZrPP})^{8)}$ and Zinc chloride ${ }^{9)}$, have been used as catalysts for the fatty acid esterification in order to avoid the problems associated with the use of homogeneous catalysts ${ }^{10)}$.

Heterogeneous catalysts based on clays have received considerable attention in different chemical processes due to their environmental compatibility, low cost, selectivity, thermal stability and recyclability ${ }^{11,12)}$. They are one of the most widely studied solid acid catalysts for many organic transformations ${ }^{13)}$ such as alkylation ${ }^{14,15)}$, condensation ${ }^{16)}$, dimerization $^{17)}$, isomerization ${ }^{18,19)}$, ether formation ${ }^{20)}$, transesterification reactions ${ }^{5)}$ and particularly in esterification reactions ${ }^{13)}$.
The layered aluminosilicates, have been widely investigated on account of the unique surface properties mainly surface acidity, cation exchange capacity and high surface areas. Montmorillonite clay is among various layered aluminosilicates which has caught great attention due especially of its good swelling property and high exchange capacity ${ }^{10)}$. Moreover, clays are cheap and have high physical strength possess relatively high resistance towards alkali treatment.

The most recent work was interested in the esterification of glycerol with lauric acid in the presence of clay chemically modified by the esterification reaction was performed in a continuously stirred flask reactor under atmospheric pressure for up to $8 \mathrm{~h}$. Different organo-montmorillonites (montmorillonite was modified with cetyl trimethylammonium bromide (CTAB), tetra-n-butylammonium bromide $(\mathrm{TBAB})^{10,21)}$ and tetra-n-butylammonium $\mathrm{TBMMT}^{22)}$ ) have been used as catalyst for the Lauric acid esterification. The amounts of organo-montmorillonite used were 3 mass $\%{ }^{22)}$ and 5 mass $\%{ }^{10,21)}$ related to reactant (lauric acid). Lauric acid conversions of about $80 \%{ }^{21,22 \text { ) }}$ and $71 \%{ }^{10)}$ were achieved using a molar ratio of glycerol: lauric acid 6:1.

In this work, it is proposed to study the esterification of ethylene glycol and glycerol by some typical fatty acids such as palmitic, stearic and oleic acids in the presence of clays as catalysts. The clays used are commercial montmorillonites under the names (KSF, KSF/O, KP10 and K10) and having different physicochemical properties. The cata-

\footnotetext{
* Correspondence to: Soumaya Bouguerra Neji, Laboratory of Natural Substances, Faculty of Sciences, University of Sfax, Route de Soukra Km 3, 5-3038- Sfax. TUNISIA

E-mail: soumaya_bouguerra@yahoo.fr

Accepted January 6, 2017 (received for review November 12, 2016)

Journal of Oleo Science ISSN 1345-8957 print / ISSN 1347-3352 online

http://www.jstage.jst.go.jp/browse/jos/ http://mc.manusriptcentral.com/jjocs
} 
lytic role of each major physico-chemical properties of these clays, for instance specific surface area, porosity, surface acidity was studied. The best operating conditions for the course of the reaction were optimized through a development of experiments which take into account the amount of catalyst, the reaction temperature and the molar ratio of the reactants. The effect of the fatty acid type, the length of the alcohol's carbon chain and also the reusability of the catalyst were also examined.

\section{EXPERIMENTAL PROCEDURE}

\subsection{Material}

Fatty acids [palmitic acid (hexadécanoic acid; C16:0; 99$100 \%$ ), stearic acid (octadecanoic acid; C18:0; 95\%) used in the reactions were procured from Sigma-Aldrich. Oleic acid (cis-9-octadecenoic acid, C18:1; 99\%)] and the four commercials clays (Montmorillonite KSF/0 clay, Montmorillonite KSF clay, Montmorillonite KP10 clay, and Montmorillonite K10 clay)were purchased from Fluka. The relevant physicochemical properties of the catalysts used are given in Table $1^{23)}$. Glycerol (1, 2, 3-propanetriol, $\left.\geq 99.5 \%\right)$ and hexane were purchased from Panreac. Ethylene glycol(1, 2-dihydroxyethane) was purchased from Labochimie. All these chemicals were used as received without any further purification.

\subsection{Esterification reaction}

The esterification reaction is carried out in a continuous reactor composed of a three-neck flask $(100 \mathrm{~mL})$ equipped with a water-cooler condenser, a thermometer and a magnetic stirrer ${ }^{13)}$.

Two grams of fatty acid (stearic, oleic or palmitic acid) and a mass of polyol (ethylene glycol or glycerol)were introduced into the reactor and were esterified in the presence of catalyst clays (KSF, KSF/O, KP10 and K10) representing $10 \%$ by weight of fatty acid. The reactions are performed at a temperature set at $150^{\circ} \mathrm{C}$.

Samples were withdrawn to determine the remaining amount of fatty acid by titration with a sodium hydroxide solution $\left(0.017 \mathrm{molL}^{-1}\right)$. The conversion of fatty acid was calculated by the following formula ${ }^{24)}$ :

$$
R \%=\frac{a_{i}-a_{t}}{a_{i}} \times 100
$$

Where $a_{i}$ is the initial acidity of the mixture and at is the final acidity at a time " $\mathrm{t}$.

\subsection{Reaction conditions}

Several reaction conditions were optimized, for example catalyst loading from $0.1 \mathrm{~g}$ to $1.2 \mathrm{~g}$, which corresponds to a mass ratio of $0.05-0.6 \mathrm{w} / \mathrm{w}$. The reaction temperatures were studied from $100^{\circ} \mathrm{C}$ to $170^{\circ} \mathrm{C}$. The molar ratios of polyol fatty acid were 1:0.5, 1:1, 1:2, 1:3 and 1:4. The type of the fatty acid and the length of the alcohol's carbon chain have also been studied. At the end of the reaction, the catalyst was separated by centrifugation and the contents in the reaction product (TG, DG, MG, and FA) were analyzed using a gas chromatograph (GC).

\subsection{Reusability study}

In the reusability study of the KSF catalyst, $10 \mathrm{wt} \%$ of fresh catalyst, with respect to the initial reactants, was used for the reaction performed at $150^{\circ} \mathrm{C}$ for $5 \mathrm{~h}$. After the experiment, the catalyst was filtered from the catalytic reaction mixture, successively washed with hexane and then dried in an oven at $80^{\circ} \mathrm{C}$. Afterwards, the catalyst was reused for the subsequent esterification runs, using the same reaction conditions. Such reusability experiments were repeated for 5 times and the activity was measured based on the conversion of oleic acid.

\section{RESULTS AND DISCUSSION}

\subsection{Catalytic activity of acid clays}

Montmorillonite is a member of the smectite clay family and has 2:1structure i.e. octahedral layer of $\mathrm{Al}$ is sandwiched between two tetrahedral layers of silicon coordinated with oxygen (TOT), exhibiting overall weak acidity ${ }^{25)}$. The crystalline sheets of negatively charged aluminosilicates are balanced by hydrated cations $\left(\mathrm{Na}^{+}, \mathrm{K}^{+}\right.$or $\left.\mathrm{Ca}^{2+}\right)$ in the interlayer spaces of montmorillonite. The most interesting features of the smectite clay are their intercalation, swelling and cation exchange capacity which improve the catalytic properties of smectite clay. In order to select the

Table 1 Some characteristic data of the investigated catalysts ${ }^{23)}$.

\begin{tabular}{lccccc}
\hline & \multicolumn{2}{c}{ Textural characteristics } & \multicolumn{2}{c}{ Acidity } \\
\cline { 2 - 6 } Catalysts & $\begin{array}{c}\text { Specific } \\
\text { surface }\left(\mathrm{m}^{2} / \mathrm{g}\right)\end{array}$ & $\begin{array}{c}\text { Average pore } \\
\text { diameter }(\AA)\end{array}$ & $p H$ & \multicolumn{2}{c}{ Surface acidity $\left(\mathrm{mmol} \mathrm{g}^{-1}\right)$} \\
\cline { 5 - 6 } $\mathrm{KSF} / \mathrm{O}$ & 117 & 74 & 1.3 & 1.03 & 0.20 \\
$\mathrm{KSF}$ & 30 & 50 & 1.5 & 0.59 & 0.15 \\
$\mathrm{KP} 10$ & 169 & 71 & 1.8 & 0.49 & 0.09 \\
$\mathrm{~K} 10$ & 249 & 56 & 4.5 & 0.33 & 0.29 \\
\hline
\end{tabular}


best clays, a series of montmorillonite KSF, KSF/0, KP10, and K10 were used as acid catalysts. Figure 1 shows the conversion profiles relative to the reaction times of oleic acid with ethylene glycol over the studied catalysts (10 wt. \%) at $150^{\circ} \mathrm{C}$. It can be noticed that the reaction rate increases according to the following order: $\mathrm{KSF} / 0>\mathrm{KSF}>$ $\mathrm{KP} 10>\mathrm{K} 10$. Acid treatment in commercial-type montmorillonite $\mathrm{KSF} / 0, \mathrm{KSF}, \mathrm{KP} 10$ and $\mathrm{K} 10$ promoted an increase in the number of acid sites of moderate strength compared to natural $\mathrm{clay}^{26)}$. The acid strength of the catalysts can be characterized by the $\mathrm{pH}$-value. The number of sites by the intensities of the Brønsted- and Lewis-characteristic bands in the IR spectra after pyridine adsorption as previously described (Table 1$)^{23}$. The acid strength increases from $\mathrm{K} 10$ to KP10 then KSF and KSF/O. It is noted that a relatively high conversion of oleic acid with ethylene glycol in the case of KSF and KSF/O. A slight decrease from(90\%) to $(83.33 \%)$ efficiency is achieved with KP10. The lowest yield was obtained with $\mathrm{K} 10$ which has the highest $\mathrm{pH}$.

The increase of the catalytic conversion could be correlated to the total acidity surface of the catalyst. Besides, lower $\mathrm{pH}$ clay values resulted in significant increase of the reaction rate. Both $\mathrm{KSF}$ and $\mathrm{KSF} / \mathrm{O}$ with initial $\mathrm{pH}$ of 1.5 and 1.3, respectively, led to higher conversion. Whereas, only $30 \%$ oleic acid conversion yield was observed for K10 which have the higher surface area $\left(249 \mathrm{~m}^{2} / \mathrm{g}\right)$ and the higher $\mathrm{pH}(4.5)$. Consequently, the catalytic activity of these K-catalysts is controlled by their acidity. The other physical properties of clays, including surface area and the average pore diameter, do not appear to have a significant effect on the variation of the yield of the reaction ${ }^{27}$. This result is in good agreement with the report of Pushpaletha et al. on the correlation between surface properties and catalytic activity of montmorillonite catalysts ${ }^{28)}$.

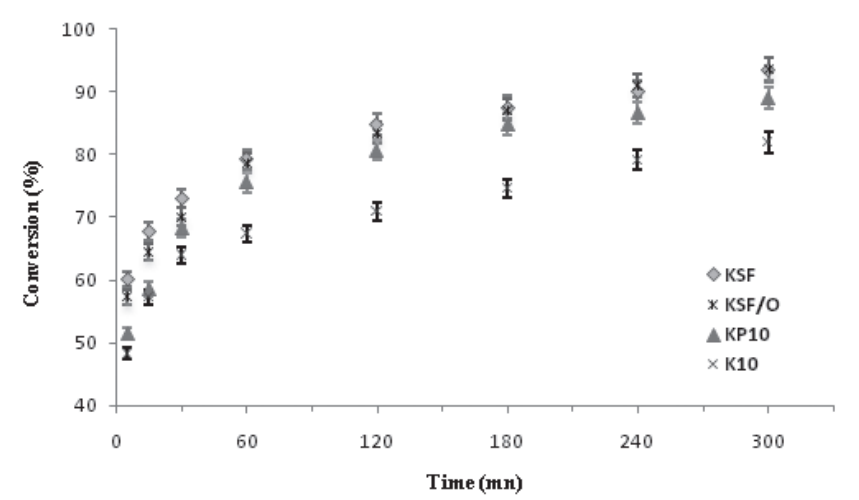

Fig. 1 Conversion profiles of the esterification of oleic acid with ethylene glycol over different acidic clay catalysts. Experiments were carried out with 1:2 oleic acid: ethylene glycol molar ratios and 10 wt. \% of catalyst at $150^{\circ} \mathrm{C}$.

\subsection{Optimization of reaction parameters}

Given the importance of the catalyst in the course of the reaction, it is necessary to study its loading.

3.2.1 Optimization of catalyst loading

Oleic acid was esterified with ethylene glycol at different weight of KSF. All experiments are carried out at $150^{\circ} \mathrm{C}$, for $5 \mathrm{~h}$. Figure 2 presents the fatty acid conversion as a function of catalyst concentration $\mathrm{Cc}$ (where $\mathrm{Cc}$ is the weight of catalyst/initial weight of fatty acid) which shows two different domains. In the first part, the ester conversion increases proportionally with the Cc. In fact, when the amount of the catalyst increases, the total number of active catalytic sites increases in the reaction medium. Beyond $0.2 \mathrm{w} / \mathrm{w}$ Cc, yields remain substantially invariant to the quantity of catalyst in excess (90\% of fatty acid was converted).

\subsubsection{Optimization of temperature reaction}

In general, the chemical reaction rate was strongly dependent on the reactor temperature. To study the temperature effect, $2 \mathrm{~g}$ of oleic acid and $0.869 \mathrm{~g}$ of ethylene glycol are esterified with $0.1 \mathrm{w} / \mathrm{w}$ KSF at different temperatures ranging from $100^{\circ} \mathrm{C}$ to $170^{\circ} \mathrm{C}$. Esterification yields obtained after 5 hours of reaction are represented as a function of temperature in Fig. 3.

It can be observed that the rate of reaction increases with temperature, especially the conversion in the range $100-150^{\circ} \mathrm{C}$. It is found that the yields vary almost linearly with temperature. This effect shows that by increasing the temperature the mobility of the molecules increases. Therefore, molecules can reach the inner pores of the catalyst more easily. After $150^{\circ} \mathrm{C}$ the yield increases but in a less important way. In the same context, Kotwal et al. also found in the esterification of fatty acids (lauric, myristic, stearic and oleic acids) with glycerol in the presence of solid catalyst Fe_Zn Double metal cyanide (DMC) has a temperature major effect on the conversion and product

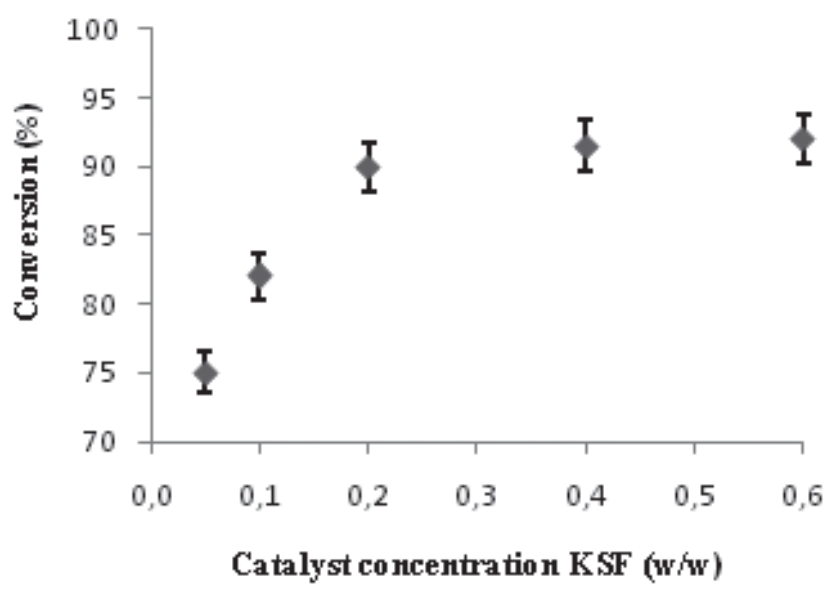

Fig. 2 Conversion(\%) of esterification of oleic acid with ethylene glycol over KSF catalyst versus catalyst concentration at $150^{\circ} \mathrm{C}$. 


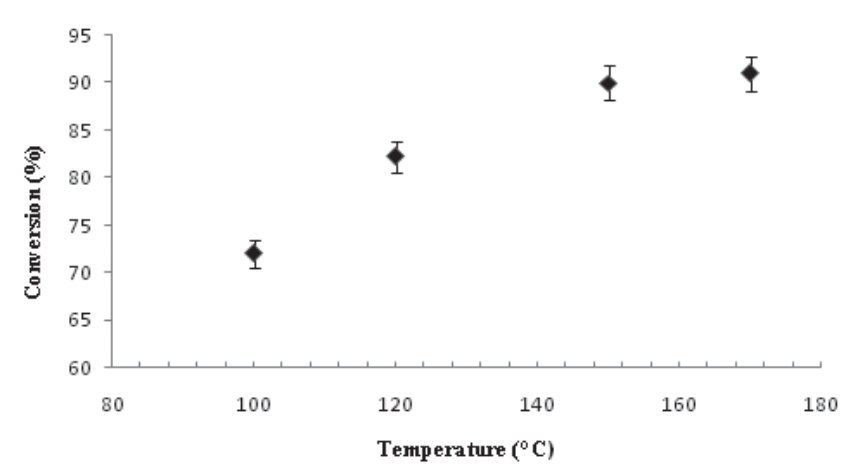

Fig. 3 Effect of reaction temperature on the yield of the esterification reaction.

selectivity. They showed that the conversion of the fatty acid is not significant below $130^{\circ} \mathrm{C}^{7)}$. The next studied parameter was the molar ratio (polyol: fatty acid).

\subsubsection{Optimization of Molar Ratio}

In this part, all experiments were carried out at the choice temperature of $150^{\circ} \mathrm{C}$. The esterification process was conducted at 1:0.5, 1:1, 1:2, 1:3 and 1:4 oleic acid to ethylene glycol. The effect of the initial concentration ratio of reactants on the reaction rate is significant. As shown in Fig. 4, the increasing molar ratio of oleic acid / ethylene glycol from 1:0.5 to 1:2 led to an increase in the esterification yields. The oleic acid conversion reached up to $93.5 \%$. In fact, more ethylene glycol was available for effective contact with the relatively more viscous oleic acid. The effect of molar ratio on the oleic acid conversion was insignificant when the oleic acid / ethylene glycol molar ratio was increased from 1:2 to 1:4. The excess of ethylene glycol beyond (1:2) molar ratio had no effect on the reaction yield. The same interpretation was deduced over the synthesis of phytosterol esters ${ }^{29)}$. This behavior can be due to a significant dilution of the oleic acid, the limiting reagent in this reaction.

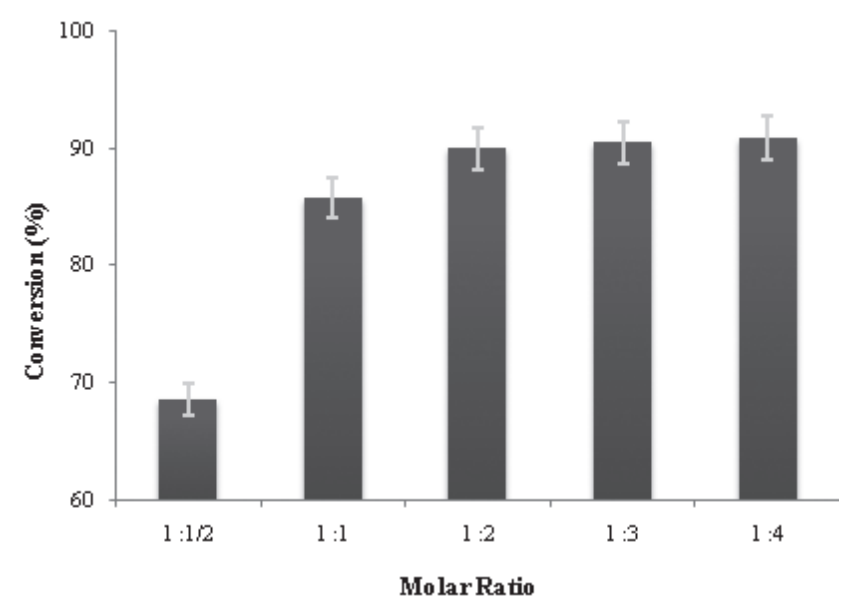

Fig. 4 Effect of oleic acid: ethylene glycol molar ratio on the esterification reaction at $150^{\circ} \mathrm{C}$.
The molar ratio is a very interesting parameter which affects not only the conversion rate, but also the glycerides composition of the mixture product can be changed. As shown in Fig. 5, an equimolar ratio of acid and glycerol can product $41.8 \%$ of diglyceride and $9.7 \%$ of triglyceride. Increasing the glycerol (1:2) leads to a high selectivity for the monoglycerides formation 43.5\%. However, (1:3) molar ratio leads to a decrease in the yield of esters and therefore decreased percentages in mono and diglycerides, the percentage of triglyceride remaining invariant.

To generalize this process, it is important to study the esterification of others fatty acids with ethylene glycol and glycerol.

\subsection{Esterification of other fatty acids}

Montmorillonite KSF was used as catalyst in the esterification of oleic, stearic and palmitic acids with ethylene glycol and glycerol. Figure 5 shows the conversion of various fatty acids with ethylene glycol and glycerol.

3.3.1 Effect of the length of the alcohol's carbon chain

As shown in Fig. 6, it was found that the yield of the esterification of fatty acid with ethylene glycol is always higher than that of the esterification of glycerol. This can

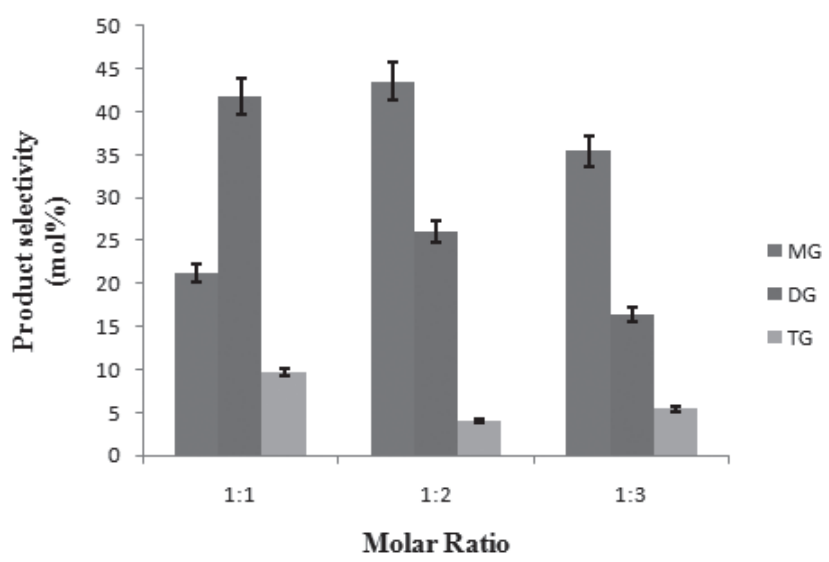

Fig. 5 Effect of glycerol: oleic acid molar ratio on the composition of esters at $150^{\circ} \mathrm{C}$.

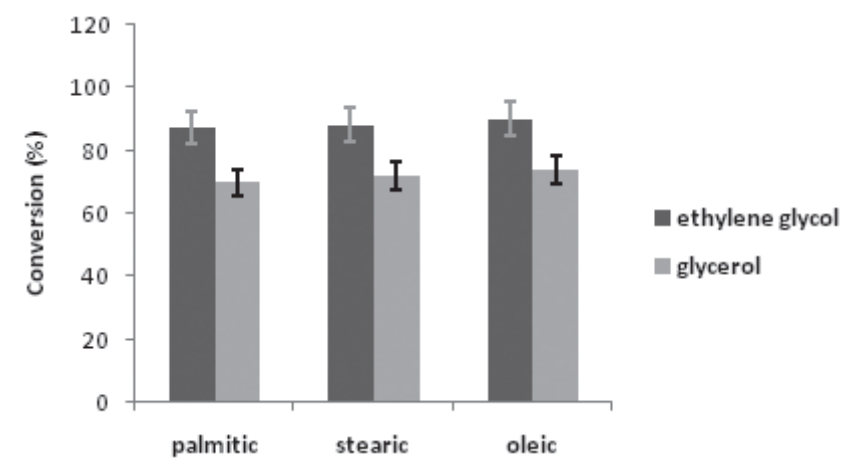

Fig. 6 Conversion profiles of the esterification of palmitic, stearic and oleic acid with ethylene glycol and with glycerol at $150^{\circ} \mathrm{C}$. 
be attributed to the high viscosity of glycerol by the contribution to that of ethylene glycol which decreased the number of shock with the fatty acid chains. Glycerol is more voluminous than ethylene glycol, its accessibility to acid functions will consequently lower. Moreover, the glycerol has two types of primary alcohols at the end and a secondary in the center, whereas ethylene glycol has only two primary alcohols. It is known that the esterification yields with a primary alcohol are greater than those obtained with secondary alcohol.

3.3.2 Effect of the type of fatty acids on the esterification yield

Figure 6 shows a slight increase in conversion to oleic acid (C18: 1). It can be concluded that any increase in hydrocarbon chain length of the fatty acid promotes an increase in the conversion rate, as indicated by the yield values of palmitic acid and stearic acid esterified with glycerol and by ethylene glycol.

The presence of unsaturation in the skeleton of the fatty acid facilitates the course of the esterification reaction by increasing the conversion rate. Oleic acid is an advantage to other fatty acids by its chain length and its double bond. This interpretation is also checked by Kotwal et al. who found, during the esterification of lauric acid (C12: 0), myristic acid (C14: 0), stearic acid (C18: 0) and oleic acid (C18: 1) with glycerol, that the chain length of the fatty acid and the existence of unsaturation have a significant effect on the conversion products and the higher efficiency is obtained with oleic acid $(\mathrm{C} 18: 1)^{7}$.

\subsection{Catalyst stability and reusability}

In the reusability study of the catalyst, $10 \mathrm{wt} . \%$ of fresh catalyst with respect to the initial reactants was used for the reaction performed at $150^{\circ} \mathrm{C}$ for $5 \mathrm{~h}$ and the ethylene glycol /Oleic acid molar ratio was set at 1:2. After the experiment, the catalyst was filtered from the catalytic reaction mixture, washed with hexane and then dried in an oven at $80^{\circ} \mathrm{C}$. Thereafter, the catalyst was reused for the subsequent esterification runs using the same reaction conditions. Such reusability experiments were repeated for 5 times. The yields of the esterification reaction during five cycles, according to the general procedure (described in the Experimental section), are presented in Fig. 7.

As seen in Fig. 7, no significant loss of activity was observed during the transesterification of oleic acid with ethylene glycol in the first 5 runs. The montmorillonite KSF catalyst showed rather good activity retention in several cycles of usage. The catalyst retained up to $96 \%$ from its initial conversion of $93.26 \%$.

\section{CONCLUSIONS}

The esterification of fatty acid with polyol over acid clays

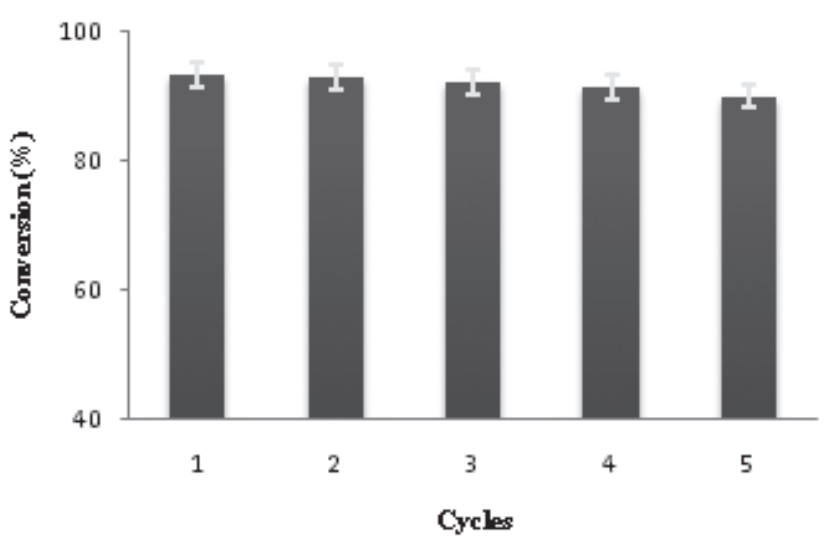

Fig. 7 Catalytic activity of the esterification of oleic acid with ethylene glycol, in five consecutive experiments.

was efficiently carried out in a continuous reactor. The catalytic activity of these K-catalysts is controlled by their acidity. It is found that the catalytic activity of clay is mainly related to the $\mathrm{pH}$ value. The other physical properties of clays, including surface area and the average pore diameter, do not appear to have a significant effect on the variation of the yield of the reaction. The montmorillonite KSF has proven to be the most effective of the tested catalysts.

Besides the catalytic activity of the catalyst type, the principal parameters affecting the reaction rate especially catalyst loading, temperature and molar ratio oil/alcohol were optimized. The best conversion achieved $(74.5 \%$ for an initial $31 \%$ acid) were obtained in the esterification of oleic acid and ethylene glycol with a molar ratio(1: 2) in a closed reactor heated at a temperature of $150^{\circ} \mathrm{C}$ in the presence of $10 \%$ by mass with respect to KSF the amount of fatty acid.

This procedure was generalized by the application of the esterification of other fatty acids, like palmitic and stearic acids, with glycerol. The yield of the esterification of fatty acid with ethylene glycol is always higher than that of the esterification of glycerol. This can be attributed to the high viscosity and volumineusty of glycerol which decreased the number of shock with the fatty acid chains. On the other hand, Oleic acid is an advantage to other fatty acids owing to its chain length and double bond.

The study of the reusability of the catalyst showed a very good stability of KSF clay which can be reused for 5 runs without significant loss in the catalytic activity. Indeed, the catalyst retained up to $96 \%$ from its initial activity after 5 consecutive cycles without any treatment.

\section{References}

1) Douglas, G.H.; Erdogan, G. Formation of polyol-fatty 
acid esters by lipases in reverse micellar media douglas. Biotechnol. Bioeng. 40, 110-118(1992).

2) Reddy, C.R.; Iyengar, P.; Nagendrappa, G.; Jai Prakash, B.S. Esterification of succinic anhydride to di- (p-cresyl) succinate over Mn+-montmorillonite clay catalysts. Mol. Catal. A Chem. 229, 31-37(2005).

3) Chen, X.; Xu, Z.; Okuhara, T. Liquid phase esterification of acrylic acid with 1-butanol catalyzed by solid acid catalysts. Appl. Catal. A Gen. 180, 261-269 (1999).

4) Abro, S.; Pouilloux, Y.; Barrault, J. Selective synthesis of monoglycerides from glycerol and oleic acid in the presence of solid catalysts. in Heterogeneous Catalysis and Fine Chemicals IV, pp. 539-546 (1997).

5) Sani, Y.M.; Daud, W.M.A.W.; Abdul Aziz, A.R. Activity of solid acid catalysts for biodiesel production: A critical review. Appl. Catal. A: Gen. 470, 140-161 (2014).

6) Wee, L.H.; Lescouet, T.; Fritsch, J.; Bonino, F.; Rose, M.; Sui, Z.; Garrier, E.; Packet, D.; Bordiga, S.; Kaskel, S.; Herskowitz, M.; Farrusseng, D.; Martens, J. A. Synthesis of monoglycerides by esterification of oleic acid with glycerol in heterogeneous catalytic process using tin-organic framework Catalyst. Catal. Lett. 143, 356363 (2013).

7) Kotwal, M.; Deshpande, S.S.; Srinivas, D.. Esterification of fatty acids with glycerol over Fe-Zn doublemetal cyanide catalyst. Catal. Commun. 12, 13021306 (2011).

8) Varhadi, P.; Kotwal, M.; Srinivas, D. Zirconium phenyl phosphonate phosphite as a highly active, reusable, solid acid catalyst for producing fatty acid polyol esters. Appl. Catal. A Gen. 462-463, 129-136 (2013).

9) Mostafa1, N.A.; Maher, A.; Abdelmoez, W. Production of mono-, di-, and triglycerides from waste fatty acids through esterification with glycerol. Adv. Biosci. Biotechnol. 4, 900-907 (2013).

10) Wibowo, T.Y.; Zakaria, R.; Abdullah, A.Z. Selective glycerol esterification over organomontmorillonite catalysts. Sains Malays. 39, 811-816 (2010).

11) Zatta, L.; Ramos, L.P.; Wypych, F. Acid-activated montmorillonites as heterogeneous catalysts for the esterification of lauric acid acid with methanol. Appl. Clay Sci. 80-81, 236-244(2013).

12) Reddy, C.R.; Lyengar, P.; Nagendrappa, G.; Jai Prakash, B.S. Esterification of dicarboxylic acids to diesters over $\mathrm{M}^{\mathrm{n}+}$-montmorillonite clay catalysts. Catal. Lett. 101, 87-91 (2005).

13) Bouguerra, N.S.; Trabelsi, M.; Frikha, M.H. Esterification of fatty acids with short-chain alcohols over commercial acid clays in a semi-continuous reactor. Energ. 2, 1107-1117(2009).

14) Kaur, M.; Sharma, S.; Bedi, P.M.S. Silica supported Brönsted acids as catalyst in organic transformations: A comprehensive review. Chin. J. Catal. 36, 520-549
(2015).

15) Adriano, L.S.N.; Tito, L.M.Z.; Angélicab, R.S.; Costa, C.E.F.; Zamiana, J.R.; Filhoa, G.N.R. Esterification of oleic acid over solid acid catalysts prepared from Amazon flint kaolin. Appl. Catal. B Environ. 101, 495-503 (2011).

16) Miranda, R.; Valencia V.O.; Abel Maya,V.C.; Nicolás, V.I.; Vargas, R.Y.M.; Morales, S.J.A.; García, R.E.; Salmón, M. Synthesis of cycloveratrylene macrocycles and benzyl oligomers catalysed by bentonite under microwave/ infrared and solvent-free conditions. Molecules 18, 12820-12844(2013).

17) Fási, A.; Pálinkó, I.; Gömöryc, Á.; Kiricsi, I. Ring opening, dimerisation and oligomerisation reactions of methyloxirane on solid acid and base catalysts. J. Mol. Catal. A Chem. 208, 307-311 (2004).

18) Wiedemann, S.C.C.; Stewart, J.A.; Soulimani, F.; Bergen-Brenkman, T.; Langelaar, S.; Wels, B.; Peinder, P.; Bruijnincx, P.C.A.; Weckhuysen, B.M. Skeletal isomerisation of oleic acid over ferrierite in the presence and absence of triphenylphosphine: Pore mouth catalysis and related deactivation mechanisms. J. Catal. 316, 24-35 (2014).

19) Perissinotto, M.; Lenarda, M.; Storaro, L.; Ganzerla, R. Solid acid catalysts from clays: acid leached metakaolin as isopropanol dehydration and 1-butene isomerization catalyst. J. Mol. Catal. A Chem. 121, 103-109 (1997).

20) Lei, L.; Plank, J. Synthesis and properties of a vinyl ether-based polycarboxylate superplasticizer for concrete possessing clay tolerance. Ind. Eng. Chem. Res. 53, 1048-1055 (2014).

21) Wibowo, T.Y.; Abdullah, A.Z.; Zakaria, R. Organo-montmorillonites as catalysts for selective synthesis of glycerol monolaurate. Appl. Clay. Sci. 50, 280-281 (2010).

22) Hashemizadeh, I.; Abdullah, A.Z. Influence of process conditions on glycerol esterification catalyzed by tetra-n-butylammonium- modified montmorillonite catalyst. J. Sci. Tech. 2, 47-51 (2012).

23) Vodnár, J.; Farkas, J.; Békássy, S. Catalytic decomposition of 1,4-diisopropylbenzene dihydroperoxide on montmorillonite-type catalysts. Appl. Catal. A Gen. 208, 329-334 (2001).

24) Carmo, A.C.; De Souza, L.K.C.; Da Costa, C.E.F.; Longo E.; Zamian, J.R.; Da Rocha F.G.N. Production of biodiesel by esterification of palmitic acid over mesoporous aluminosilicate Al-MCM-41. Fuel 88, 461-468 (2009).

25) Jha, A.; Garade, A.C.; Shirai, M.; Rode, C.V. Metal cation-exchanged montmorillonite clay as catalysts for hydroxyalkylation reaction. Appl. Clay Sci. 74, 141146 (2013).

26) Bieseki, L.; Treichel, H.; Araujo, A.S.; Pergher, S.B.C. Porous materials obtained by acid treatment process- 
ing followed by pillaring of montmorillonite clays. Appl. Clay Sci. 85, 46-52 (2013).

27) Bouguerra Neji, S.; Azabou, S.; Contreras, S.; Medina, F.; Bouaziz, M.; Novel mild synthesis of high-addedvalue $p$-hydroxyphenyl acetic acid and 3,4-dihydroxyphenyl acetic acid using the acidic clay/hydrogen peroxide catalytic system. C. R. Chimie 19, 1-7 (2016).

28) Pushpaletha, P.; Rugmini, S.; Lalithambika, M. Correlation between surface properties and catalytic activity of clay catalysts. Appl. Clay Sci. 30, 141-153(2005).

29) Zheng, M.; Dong, L.; Lu, Y.; Guo, P.M.; Deng, Q.C.H.; Li, W.L.; Feng, Y.Q.; Huang, F.H. Immobilization of Candida rugosa lipase on magnetic poly (allyl glycidyl ether-co-ethylene glycol dimethacrylate) polymer microsphere for synthesis of phytosterol esters of unsaturated fatty acids. J. Mol. Catal. B Enzym. 74, 16-23 (2012). 